\title{
GÊMEOS SIAMESES: UM RELATO DE CASO
}

\section{ARTIGO ORIGINAL}

SOUZA, Izabella Amorim de ${ }^{1}$

SILVEIRA, Letícia Magalhães ${ }^{2}$

CARVALHO, Morisa Martins Leão ${ }^{3}$

SOUZA, Izabella Amorim de. SILVEIRA, Letícia Magalhães. CARVALHO, Morisa Martins Leão. Gêmeos siameses: um relato de caso. Revista Científica Multidisciplinar Núcleo do Conhecimento. Ano 05, Ed. 08, Vol. 06, pp. 106-115. Agosto de 2020. ISSN: 2448-0959, Link de acesso: https://www.nucleodoconhecimento.com.br/saude/gemeos-siameses

\section{RESUMO}

Gemelares imperfeitos são raros, mas de grande interesse para obstetras. O diagnóstico precoce por ultrassonografia pode facilitar prognóstico gestacional, a via de parto e o planejamento pós-natal. Dito isso, o presente artigo tem por objetivo relatar um caso de gemelaridade imperfeita, de gemelares unidos, com 24 semanas e 5 dias, com anomalias estruturais exuberantes demonstradas pelos exames de imagem. Utilizando como metodologia à pesquisa bibliográfica (portais como PubMed, SciELo e Lilacs), com descritores "gêmeos siameses, gemelaridade imperfeita, gêmeos unidos, gestação gemelar"; relato de caso de gemelares unidos, com várias anomalias estruturais e discussão de suas características externas e achados de acordo com exames de imagem realizados. Apresentando como resultado uma

\footnotetext{
${ }_{1}^{1}$ Discente do curso de Medicina da Universidade Brasil de Fernandópolis - SP, Brasil. 2 Discente do curso de Medicina da Universidade Brasil de Fernandópolis - SP, Brasil. ${ }^{3}$ Orientadora. Mestrado em Mestrado em Bioengenharia. Especialização - Residência médica. Especialização em Especialização em Reprodução Assistida. Especialização em Pós-graduação Latu-Sensu em Écografia em G.O. Graduação em Medicina.
} 
gestante com antecedentes obstétricos Gl, P0, A0; exame físico e laboratoriais da rotina pré-natal normais; gestação com amniorrexe prematura (36 semanas e 5 dia); submissão à cesariana de emergência; características dos gêmeos: com peso 3,590kg, ambas do sexo feminino; gemelar 1: altura 42, PC 33,5, PT 36,5, PA 33,6; gemelar 2: altura de 41cm, PC 31,5, PT 36,5, PA 33,6; Apgar de 03/07 (primeiro minuto) para ambas; submetidos à ventilação com pressão positiva de $\mathrm{O}_{2}$ a $100 \%$. $\mathrm{Na}$ qual foi possível concluir que gemelares imperfeitos são raros e de grande interesse para obstetras em geral. O diagnóstico precoce pode facilitar o prognóstico da gestação, correta determinação da via de parto e planejamento pós-natal. Gêmeos fusionados, em sua maioria, são natimortos, ou morrem pouco tempo após o nascimento. Quando nascidos vivos, podem submeter-se à cirurgia de separação, dependendo do tipo de fusão que apresentam.

Palavras-chaves: Gêmeos siameses, gemelaridade imperfeita, gêmeos unidos, gestação gemelar.

\section{INTRODUÇÃO}

A gemelaridade é originada da fertilização de dois óvulos por dois espermatozoides ou da segmentação de um embrião resultante da fertilização de um óvulo por um espermatozoide. No primeiro caso, a gemelaridade é ocasionada por condições naturais e artificiais; no segundo, não depende de nenhuma condição e ocorre de forma aleatória (BEREZOWSKI et. al., 2010); (CUNNINGHAM et. al., 2005); (EGAN et. al., 2004).

A gemelaridade imperfeita, por sua vez, é uma anomalia rara, cuja etiologia ainda não foi esclarecida. Alguns autores defendem a teoria de que fatores atuantes entre o $13^{\circ}$ e $015^{\circ}$ dia após a fecundação causariam uma divisão anormal do disco embrionário levando a múltiplas formas de fusão (TEIXEIRA et. al., 2003); (FEITOSA E CASTRO et. al., 1994); (DONALDSON et. al., 1990); (POSSER; POSSER, 1996).

Gêmeos siameses são monozigóticos, sempre do mesmo sexo, tendo uma única placenta e podendo ser, mais frequentemente, monoamnióticos e, mais raramente, 
diamnióticos. Pode-se observar a incidência de anomalias relacionadas aos locais de junção e ocorrência de defeitos maiores como, por exemplo, cardiopatias congênitas, espinha bífida, higroma cístico, alterações de membros, defeitos de parede abdominal e hérnia diafragmática (DENARDIN et. al., 2013); (BRIZOT et. al., 2011).

Gêmeos fusionados podem ser classificados de acordo com o local de fusão mais proeminente em: craniópagos (crânio), toracópagos (tórax), onfalópagos (abdome), pigópagos (sacro), isquiópagos (pelve) e raquípagos (canal medular). Também se classificam em simétricos ou assimétricos (DENARDIN et. al., 2013); (UNAL et. al., 2010). Quanto mais tardia for a fusão mais incompleta será a separação e mais complexas serão as alterações (DENARDIN et. al., 2013); (SPENCER, 2000). Podese também classificá-los em ventral (união pelo abdome com umbigo único) e dorsal (união no tubo neural com abdome e cordão umbilical separados). $O$ tipo mais comum é o toracópago (67\% dos casos) (DENARDIN et. al., 2013); (MCHUGH et. al., 2006).

O diagnóstico de gemelaridade imperfeita pode ser feito no período pré-natal ou após o nascimento (DENARDIN et. al., 2013); (HILL, 1997). Ainda no pré-natal, a ultrassonografia obstétrica pode diagnosticar gêmeos acolados desde 12 semanas de gestação (DENARDIN et. al., 2013); (WEISS; DEVINE, 2002). Na primeira ultrassonografia pré-natal, fatores como uma única vesícula vitelínica extra-amniótica, embriões que se movem ao mesmo tempo e embrião com aparência bífida antes de 10 semanas de gestação sugerem gemelaridade imperfeita (DENARDIN et. al., 2013); (CUILLIER et. al., 2012).

No entanto, no final da gestação, principalmente no caso de obesidade materna ou oligoâmnio podem-se encontrar dificuldades na realização deste exame, sendo indicado, então, a ressonância nuclear magnética. Essa tecnologia é cada vez mais utilizada no diagnóstico pré-natal, principalmente devido a técnica Ultrafast, que permite imagens de alta resolução em curtos intervalos de tempo (SOUZA et. al., 2006); (CHEN; CHOE, 2003). O período ideal para sua realização é entre 24 e 40 semanas, e sua importância também se deve ao fato de auxiliar no planejamento cirúrgico pós-nascimento (DENARDIN et. al., 2013). 
A relevância do tema, as dificuldades de procedimentos e interesse de obstetras por romper esse desafio despertaram e justificaram o presente estudo, cujo objetivo foi relatar um caso de gemelaridade imperfeita.

\section{DESCRIÇÃO DO CASO}

As informações contidas neste trabalho foram obtidas por meio de revisão de prontuário, entrevista com paciente, registro fotográfico dos métodos diagnósticos aos quais a paciente foi submetida e revisão de literatura.

\section{RELATO DO CASO}

Paciente G.R.V., 21 anos, gestante de aproximadamente 24 semanas e 5 dias (ultrassonografia). Quando descobriu sua gestação, foi encaminhada ao Hospital de Base de São José do Rio Preto, por ser uma gestação gemelar imperfeita, diagnosticada por ultrassonografia em sua cidade de origem, no dia 2 de abril de 2019. Foram visualizados gêmeos com idade gestacional compatível com a biometria fetal (24 semanas e 5 dias): gestação tópica com fetos unidos a partir do tórax (gêmeos siameses), em situação longitudinal, apresentação pélvica, com dorso à direita; presença de dois membros superiores e dois membros inferiores; alteração da configuração das câmaras cardíacas. As imagens ultrassonográficas (figuras 1 a 4) documentam as principais alterações morfológicas descritas dos gêmeos acoplados. 


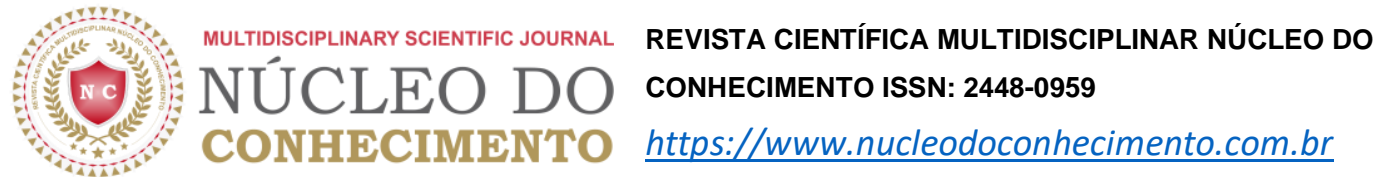

Figura 1 - Ultrassonografia demonstrando os polos cefálicos de cada gemelar.

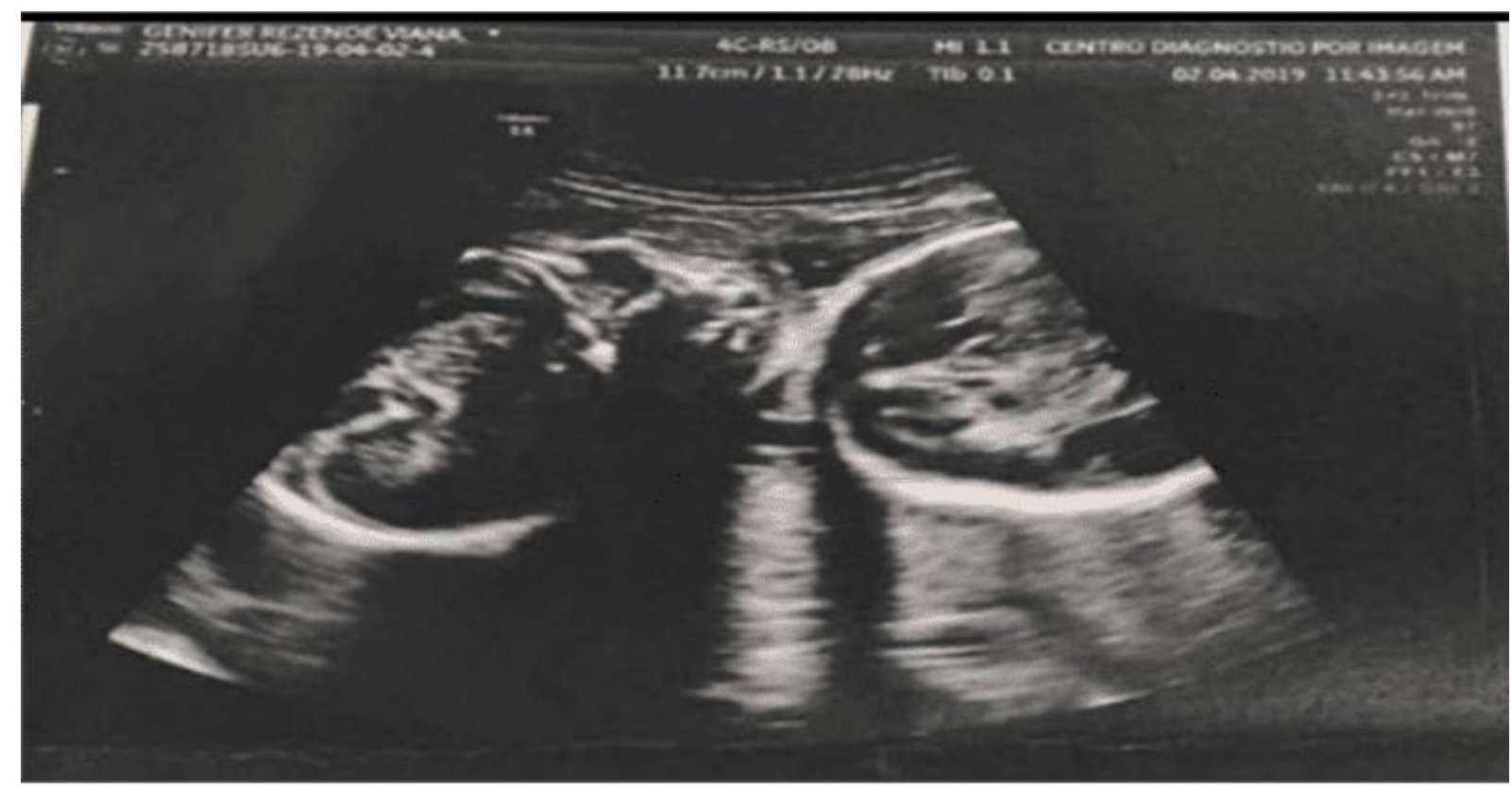

Fonte: próprio autor.

Figura 2 - Ultrassonografia demonstrando os dois estômagos de cada gemelar em uma única região abdominal.

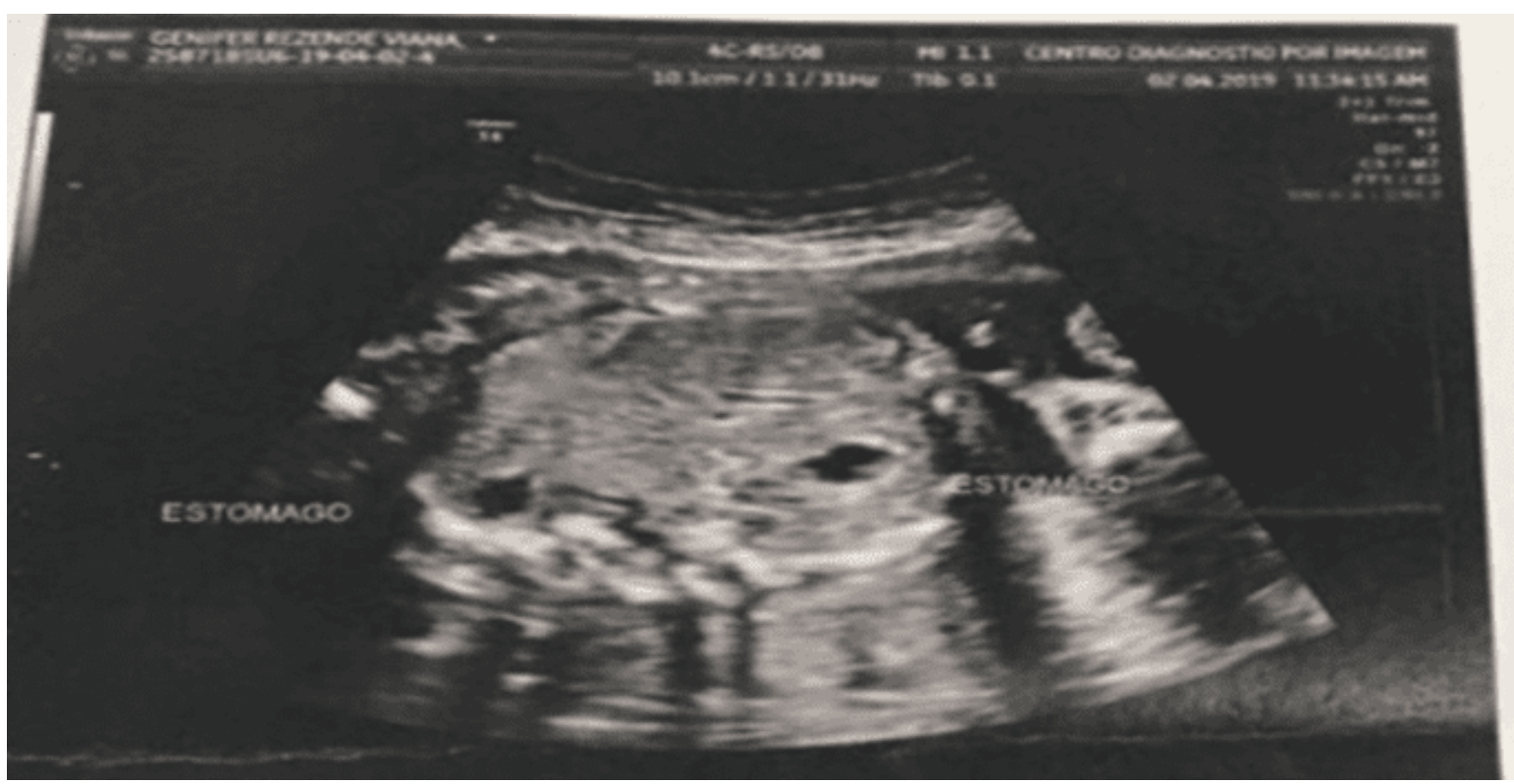

Fonte: próprio autor.

$\mathrm{RC}: 57876$

Disponível em: https://www.nucleodoconhecimento.com.br/saude/gemeos-siameses 


\section{MULTIDISCIPLINARY SCIENTIFIC JOURNAL

Figura 3 - Ultrassonografia demonstrando os gêmeos unidos pelo do tórax.

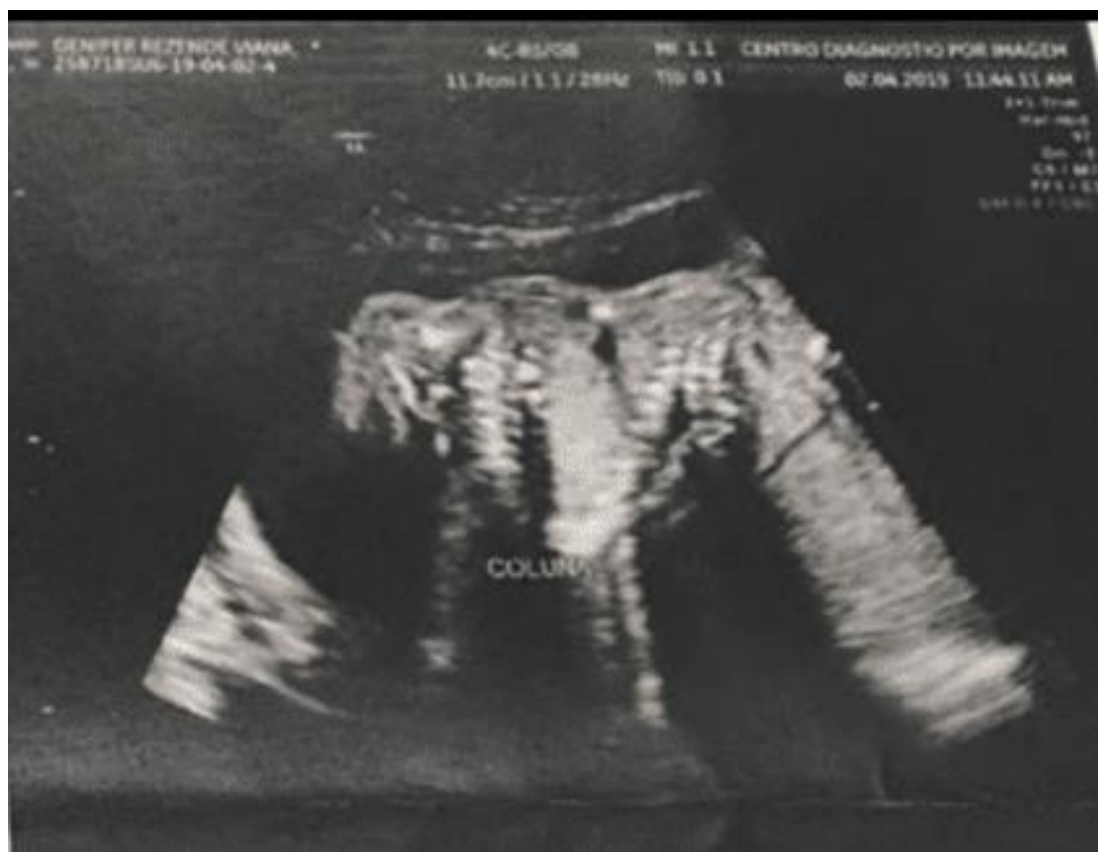

Fonte: próprio autor.

Figura 4 - Ultrassonografia demonstrando 2 tíbias e 2 fíbulas os membros inferiores dos gêmeos siameses.

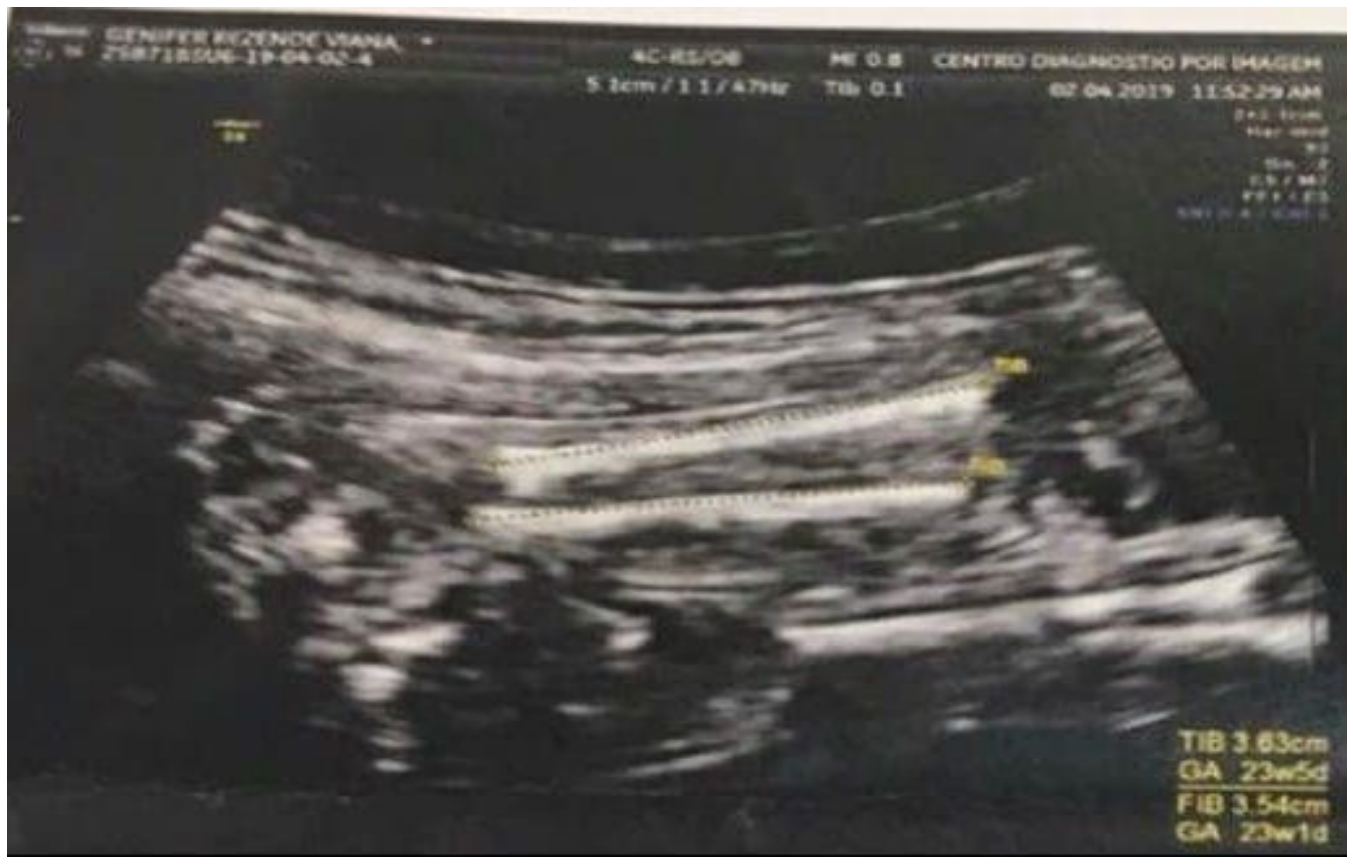

Fonte: próprio autor.

RC: 57876

Disponível em: https://www.nucleodoconhecimento.com.br/saude/gemeos-siameses 
A gestante apresentava antecedentes obstétricos $\mathrm{GI}, \mathrm{P0}, \mathrm{A0}$. O exame físico e os exames laboratoriais da rotina pré-natal foram normais (conferir), tendo iniciado o prénatal tardio. Refere que, durante todo o período gestacional, fez uso de ácido fólico e sulfato ferroso. Anteriormente ao período gestacional, refere que fazia uso de anticoncepcional. Negava doenças pregressas. Negava alergia a medicamentos. Negava realização de cirurgias. Negava etilismo, tabagismo ou uso de qualquer tipo de droga ilícita antes ou durante o período gestacional. Não há história familiar sobre caso de gemelaridade. Avó paterna hipertensa, avó materna diabética e mãe com história de transtorno depressivo.

Entretanto, com 36 semanas e 5 dias, a gestação evoluiu com ruptura prematura de bolsa, sendo submetida a cesariana de emergência em hospital na cidade de Fernandópolis. As gêmeas nasceram em apresentação pélvica às 8h58min do dia 25 de junho de 2019.

Ao exame físico, pesavam $3,590 \mathrm{~kg}$, ambas do sexo feminino. $O$ gemelar 1 , com altura de $42 \mathrm{~cm}$, PC 33,5; PT 36,5 e PA 33,6; o gemelar 2, com altura de 41cm, PC 31,5; PT 36,5 e PA de 33,6. O Apgar, no primeiro minuto, foi de 03/07 para ambas. Foram submetidas à ventilação com pressão positiva de $\mathrm{O}_{2}$ a 100\% (Figura 5). 
Figura 5 - Aspecto dos gêmeos siameses após o nascimento.

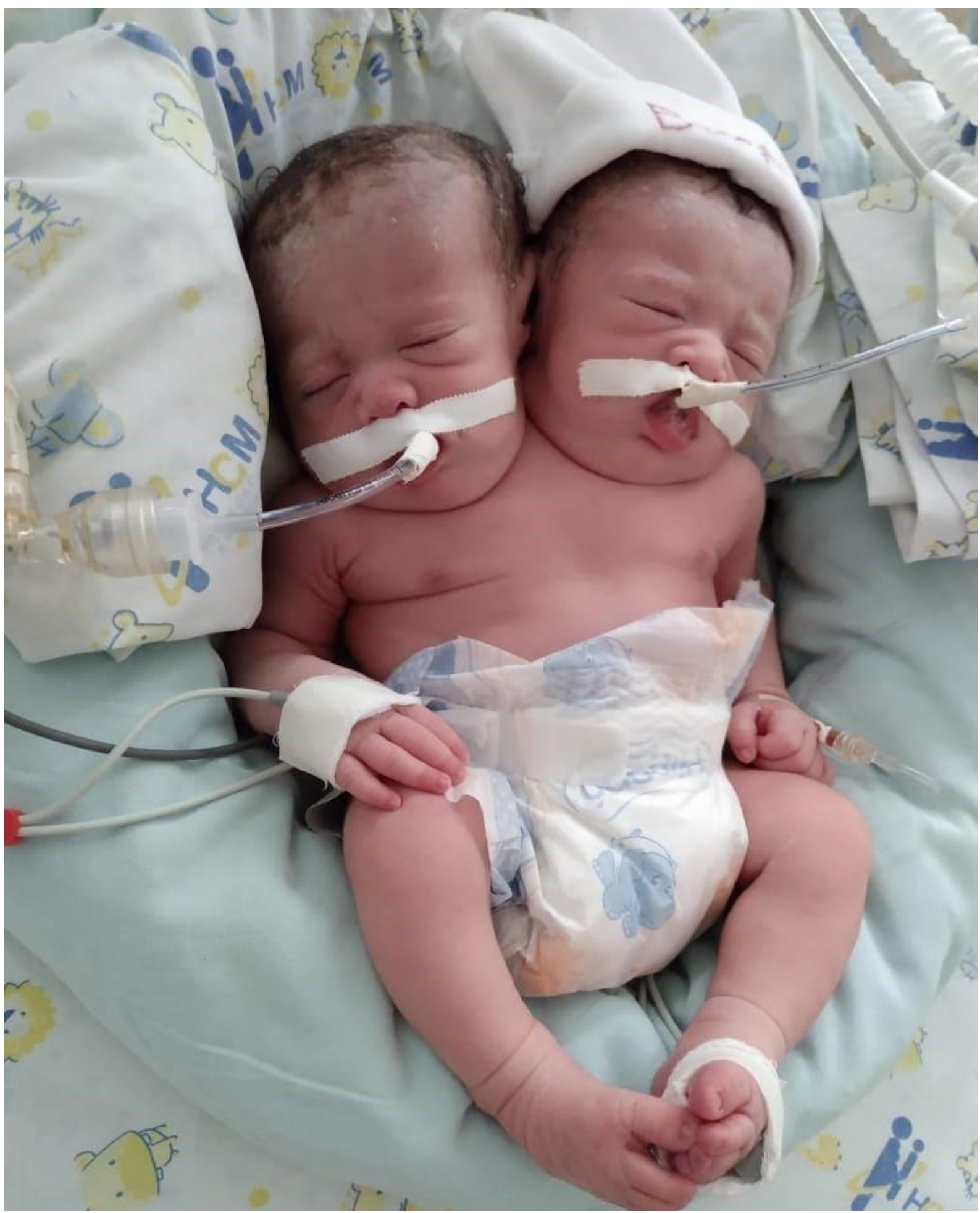

Fonte: próprio autor. 
O estudo radiológico evidenciou, pós-nascimento, gemelar siamês com fusão de arcos costais, bacia única e mediastino centrado entre as caixas torácicas (Figura 6).

Figura 6 - Estudo radiológico dos gêmeos siameses.

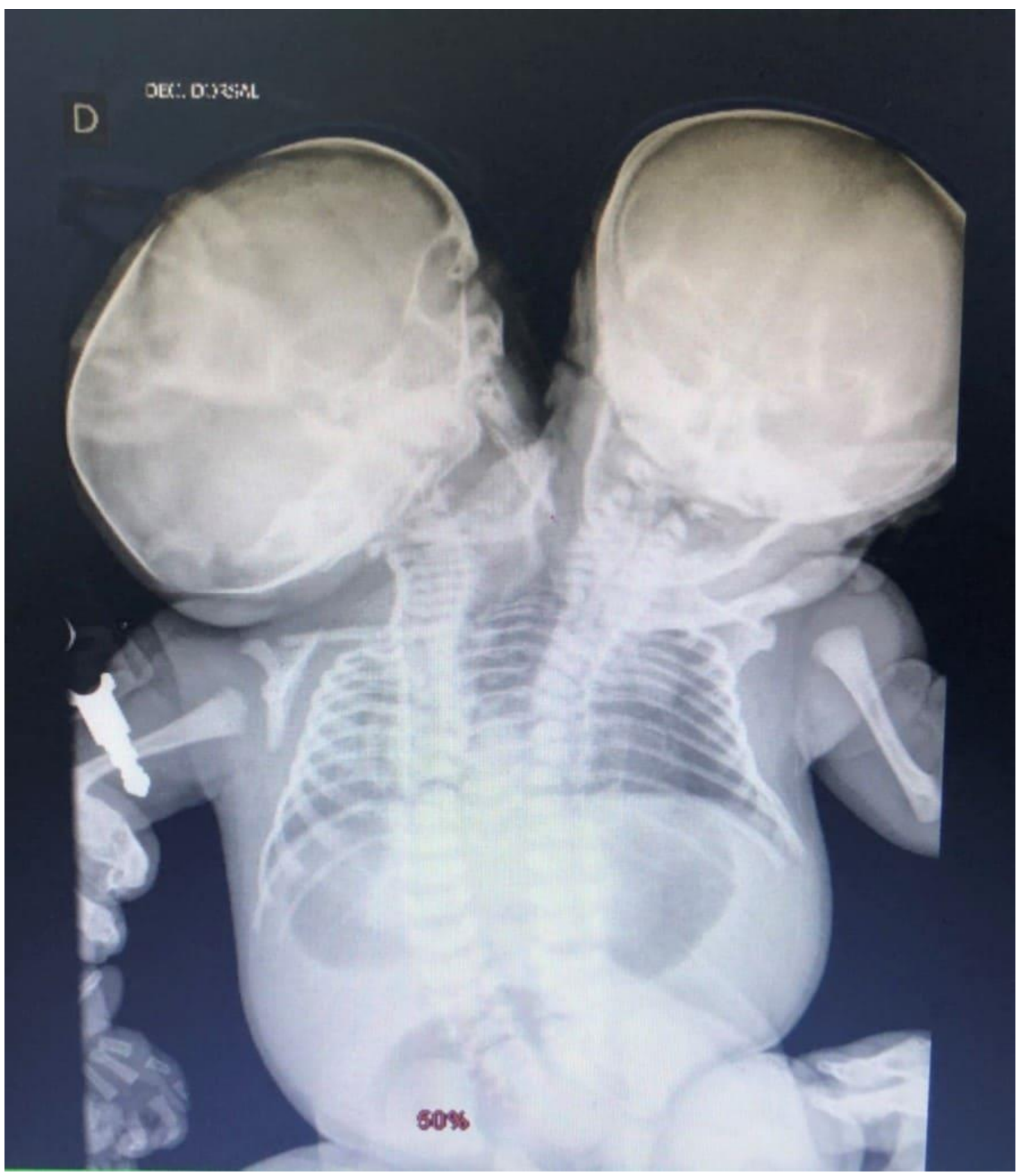

Fonte: próprio autor. 


\section{DISCUSSÃO}

A descrição do caso se deve à raridade da ocorrência de gemelaridade imperfeita. As gemelaridades múltiplas espontâneas têm incidência em 1,6\% de todas as gestações humanas, sendo 1,2\% dizigóticos e 0,4\% monozigóticos. Acredita-se que a frequência de gêmeos siameses seja em torno de 1/45.000 - 200.000 nascidos vivos. Devido ao diagnóstico precoce e posterior interrupção da gestação, a incidência de nascidos vivos com essa condição reduziu nos últimos anos (DENARDIN et. al., 2013). Assim sendo, o caso relatado neste trabalho é dito como raro (DENARDIN et. al., 2013).

Uma das causas do aumento da incidência de monocorionicidade e, consequentemente, da prevalência de gemelaridade imperfeita, refere as técnicas de reprodução assistida. Essas tecnologias aumentaram sua ocorrência em oito vezes (DENARDIN et. al., 2013). Existem duas teorias que procuram explicar esse fenômeno. A teoria da fissura, mais aceita, afirma que um único óvulo fertilizado se divide em dois embriões entre 13 e 15 dias após a fertilização (DENARDIN et. al., 2013). Por outro lado, a teoria da fusão defende que há união de dois embriões originalmente separados por volta dos 12 dias após a fertilização (DENARDIN et. al., 2013).

Gêmeos fusionados apresentam alta mortalidade, uma vez que o sucesso da cirurgia depende da complexidade da fusão, extensão da junção dos órgãos compartilhados, gravidade das anomalias e das condições clínicas dos gêmeos (DENARDIN et. al., 2013). No caso relatado, de acordo com USG obstétrico morfológico do $2^{0}$ trimestre, as gêmeas siamesas eram unidas a partir do tórax e apresentavam coração único com alteração da configuração das câmaras cardíacas. Nascidas no dia 25 de junho de 2019, as gêmeas siamesas sobreviveram até o dia 2 de julho de 2019.

\section{CONCLUSÃO}

A gemelaridade imperfeita é uma anomalia rara, de etiologia ainda não esclarecida, cujo diagnóstico pode ser realizado no pré-natal, através da ultrassonografia 
obstétrica. Quando nascidos vivos, os gêmeos siameses podem ser submetidos à cirurgia para sua separação, dependendo do tipo de fusão que apresentam. No caso relatado neste trabalho, não havia perspectiva cirúrgica devido à complexidade de junção entre as gêmeas.

\section{REFERÊNCIAS}

BEREZOWSKI, A. T; DUARTE, G; RODRIGUES, R; CAVALLI, R. C; SANTOS, R. O. C; VICENTE, Y. A. M. V. A; TAZIMA, M. F. G. S. Gêmeos conjugados: experiência de um hospital terciário do sudeste do Brasil. Rev. Bras. Ginecol. Obstet., Rio de Janeiro, fev. 2010; 32 (2): 61-65.

BRIZOT, M. L; LIAO, A. W; LOPES, L. M; OKUMURA, M; MARQUES, M.S; KREBS, $\mathrm{V}$. et al. Conjoined twins pregnancies: experience with 36 cases from a single center. Prenat Diagn 2011; 31:1120-5.

CHEN, P. L; CHOE, K. A. Prenatal MRI of heteropagus twins. AJR Am J Roentgenol. 2003 Dec.;181(6):1676-8.

CUNNINGHAM, F. G; LEVENO, K. J; BLOOM, S. L; HAUTH, J. C; GILSTRAP, III L. C; WENSTROM, K. D; Williams Obstetrics. 22 ${ }^{\text {th }}$ ed. New York: McGraw-Hill; 2005. Chapter 39, Multifetal gestation. p. 911-43.

CUILLIER, F; DILLON, K.C; GROCHAL, F; SCEMAMA, J. M; GERVAIS, T; CEREKJA, A. et al. Conjoined twins: what ultrasound may add to management. J Prenat Med 2012;6:4-6.

DENARDIN, D; TELLES, J. A. B; BETAT, R. S; FELL, P. R. K; CUNHA, A. C; TARGA, L. V; ZEN, P. R. G; ROSA, R. F. M. Gemelaridade imperfeita: um dilema clínico e ético. Revista Paulista Pediatria, São Paulo, set. 2013;31(3): 384-391.

DONALDSON, J. S; LUCK, R. S; VOGELZANG R; Preoperative CT and RM Imaging of ischiopagus twins. J Comput Assist Tomogr 1990;14:643-6. 
EGAN, J. F; BORGIDA, A. F; Multiple gestations: the importance of ultrasound. Obstet Gynecol Clin North Am. 2004;31(1):141-58.

FEITOSA E CASTRO; CUNHA, S. P; FEITOSA E CASTRO, et al. Diagnóstico prénatal de gêmeos unidos. Rev Bras Ginecol Obstet 1994;16:141-3.

HILL, L. M. The sonographic detection of early first-trimester conjoined twins. Prenat Diagn 1997;17:961-3.

MCHUGH, K; KIELY, E. M; SPITZ, L. Imaging of conjoined twins. Pediatr Radiol 2006;36:899-910.

POSSER, A. O; POSSER, Z. B. R. Gemelaridade imperfeita. Femina 1996;24:73942.

SPENCER, R. Theoretical and analytical embryology of conjoined twins: part I: embryogenesis. Clin Anat 2000;13(1):36-53.

SOUZA, A. S. R; MEDEIROS, C. C; NORONHA NETO, C; LIMA, M. M. S; LINS, G. V. Q. Diagnóstico pré-natal de gêmeos unidos com uso da ressonância nuclear magnética: relato de dois casos. Rev. Bras. Ginecol. Obstet., Rio de Janeiro JUL. 2006;28(7): 416-423.

TEIXEIRA, A. C; JULIO, H; MAZER, S; URBAN, L. A. B. D. Gemelaridade imperfeita: avaliação pelos métodos de imagem. Radiol Bras, São Paulo, jan. 2003;36(1):5760.

UNAL, O; ARSLAN, H; ADALI, E; BORA, A; YILDIZHAN, R; AVCU, S. MRI of omphalopagus conjoined twins with a Dandy-Walker malformation: prenatal true FISP and HASTE sequences. Diagn Interv Radiol 2010;16:66-9.

WEISS, J. L; DEVINE, P. C. False positive diagnosis of conjoined twins in the first trimester. Ultrasound Obstet Gynecol 2002 Nov.;20(5):516-8.

Enviado: Julho, 2020. 
Aprovado: Agosto, 2020. 